\title{
Remote ischemic preconditioning protects the spinal cord against ischemic insult: An experimental study in a porcine model
}

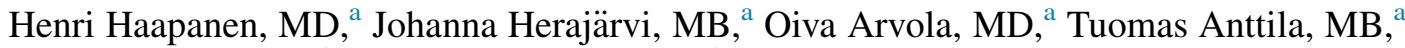 \\ Tuomo Starck, PhD, ${ }^{\mathrm{b}}$ Mika Kallio, MD, PhD, ${ }^{\mathrm{b}}$ Vesa Anttila, MD, PhD, ${ }^{\mathrm{a}}$ Hannu Tuominen, MD, PhD, \\ Kai Kiviluoma, $\mathrm{MD}, \mathrm{PhD},{ }^{\mathrm{d}}$ and Tatu Juvonen, $\mathrm{MD}, \mathrm{PhD}^{\mathrm{a}}$
}

\begin{abstract}
Objective: Surgical repair of thoracoabdominal aneurysm jeopardizes the vascularization of the spinal cord, and therefore, despite improvement in surgical techniques, still carries the risk of paraplegia. This study aimed to demonstrate the possible protective effects of remote ischemic preconditioning (RIPC) on the preservation of spinal cord function after segmental artery (SA) occlusion.
\end{abstract}

Methods: Twenty piglets were randomized into the RIPC group $(n=10)$ and the control group $(n=10)$. The RIPC group underwent transient left hind limb ischemia before systematic left subclavian artery and SA occlusion at the level of the diaphragm. Motor-evoked potential (MEP) monitoring was performed from the hind limbs. Afterward, the thoracic and lumbar spinal cords were harvested and analyzed.

Results: The elevation of the MEP amplitude after RIPC was statistically significant, whereas amplitude was consistently decreased in the control group. Additionally, the onset latency was significantly shorter after RIPC during SA occlusion. The control group reached a 50\% decrease of MEP amplitude in the right hind limb sooner than did the experimental group.

Conclusions: Remote ischemic preconditioning preserves spinal cord function after left subclavian artery and SA occlusion, as indicated by the MEP amplitudes. (J Thorac Cardiovasc Surg 2016;151:777-85)

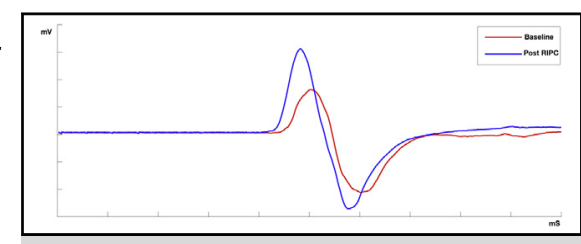

Motor-evoked potential amplitude is higher after remote ischemic preconditioning.

\section{Central Message}

Remote ischemic preconditioning preserves spinal cord function after ischemic insult, as indicated by motor-evoked potentials.

\section{Perspective}

A significant risk of paraplegia still accompanies the surgical repair of thoracoabdominal aneurysms. Studies in a clinically relevant porcine model are important to verify the safety of and refine methods designed to prevent paraplegia. Remote ischemic preconditioning is one of the most studied topics in vascular medicine. This study revealed a protective effect of this method in the spinal cord.

See Editorial Commentary page 786

See Editorial page 616.
Permanent paraplegia or paraparesis after interruption of the spinal cord blood supply is a devastating complication of thoracoabdominal aortic repair procedures, although surgical techniques and the use of adjuncts such as cerebrospinal fluid drainage, hypothermia, and pharmacologic methods have improved outcomes remarkably in recent years. ${ }^{1-4}$

\footnotetext{
From the Departments of Surgery, ${ }^{\mathrm{a}}$ Clinical Neurophysiology, ${ }^{\mathrm{b}}$ Pathology, ${ }^{\mathrm{c}}$ and Anesthesiology, ${ }^{\mathrm{d}}$ Oulu University Hospital (MRC) and University of Oulu, Oulu, Finland.

This research was supported by a grant from the Finnish Foundation for Cardiovascular Research and the Sigrid Juselius Foundation.

Received for publication June 7, 2015; revisions received July 2, 2015; accepted for publication July 12, 2015; available ahead of print Aug 20, 2015

Address for reprints: Tatu Juvonen, MD, PhD, Oulu University Hospital, Department of Surgery, P.O. Box 21, 90029 OYS, Oulu, Finland; (E-mail: tatu. juvonen@oulu.fi).

$0022-5223 / \$ 36.00$

Copyright (c) 2016 by The American Association for Thoracic Surgery

http://dx.doi.org/10.1016/j.jtcvs.2015.07.036
}

The segmental and nonsegmental vessels, primarily the subclavian and hypogastric arteries, are thought to form a vascular bed that is more crucial for spinal cord blood supply than is a single major artery arising from near the level of the diaphragm, as shown by Griepp and colleagues ${ }^{5}$ in studies of the spinal cord blood supply. The threshold for spinal cord ischemia seems to be related to the number of segmental arteries (SAs) that are occluded and thereby cause a fatal interruption to the vascular bed supply.

Scanning this QR code will take you to the article title page.

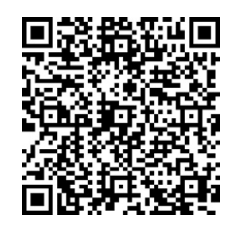




\section{Abbreviations and Acronyms \\ MEP $=$ motor-evoked potential \\ $\mathrm{RIPC}=$ remote ischemic preconditioning \\ SA $=$ segmental artery}

Two methods are available for spinal cord protection: maintenance of spinal cord perfusion during aortic clamping and neuroprotection during the time of ischemia and reperfusion. ${ }^{6}$ Attenuation of ischemic and reperfusion injuries can be achieved by priming neuronal tissue with a sublethal ischemic insult, a phenomenon known as remote ischemic preconditioning (RIPC). Thus, RIPC might be an effective way to prevent irreversible ischemic injury to the spinal cord during thoracoabdominal procedures.

The precise mechanism of RIPC has been the subject of intensive investigation. Currently, biochemical messengers, activation of the nerve pathways, or a combination of these, are considered to be protective mechanisms. ${ }^{7}$ Measuring motor-evoked potentials (MEPs) has been found to be a sophisticated and sensitive method for predicting ischemia before irreversible damage. ${ }^{2,8,9}$ A combination of these methods might provide valuable information about spinal cord protection in ischemia. Our objective was to investigate whether RIPC preserves spinal cord function after the interruption of the spinal cord blood supply in a porcine model of spinal cord ischemia with intraoperative measuring of MEPs.

\section{METHODS}

\section{Experimental Setting}

Twenty female juvenile pigs from a native stock $(19-22 \mathrm{~kg})$ were randomized into 2 groups using sealed envelopes: an RIPC group $(n=10)$ and a control group $(\mathrm{n}=10)$. RIPC was performed in the RIPC group in advance of the segmental artery (SA) occlusion. The control group underwent a protocol identical to that in the RIPC group, excluding preconditioning.

\section{Preoperative Management}

All animals received humane care in accordance with the "Principles of Laboratory Animal Care" formulated by the National Society for Medical Research and the "Guide for the Care and Use of Laboratory Animals" (http://www.nap.edu/catalog/5140.html). The animals were in the same cage at least 7 days before experimentation, and the environment was kept nonstressful. The social nature of the animals was taken into consideration preoperatively. The Research Animal Care and Use Committee of the University of Oulu approved the study.

\section{Anesthesia Protocol}

Animals were sedated with an intramuscular injection of ketamine (350 $\mathrm{mg}$ ), midazolam (45 mg), and medetomidine (1.5 mg). After endotracheal intubation, the subjects were ventilated with $50 \%$ oxygen and an end-tidal carbon dioxide concentration of $4.5 \%$ and $5.0 \%$. Anesthesia was induced with fentanyl $(50 \mu \mathrm{g} / \mathrm{kg})$ and was maintained throughout the entire experiment by a continuous infusion of fentanyl $(25 \mu \mathrm{g} /[\mathrm{kg} \cdot \mathrm{h}])$ and ketamine $(15 \mathrm{mg} /[\mathrm{kg} \cdot \mathrm{h}])$. Ketamine or fentanyl has little or no effect on evoked potentials. ${ }^{10-12}$ Sevoflurane $1.0 \%$ was used as inhalation anesthesia, which was discontinued before baseline measurements. Rocuronium $(0.1 \mathrm{mg} /$ $\mathrm{kg}$ ) was used for relaxation and was not given after the baseline values were recorded.

\section{Hemodynamic Monitoring}

An arterial line for arterial pressure monitoring and blood sampling was placed in the right femoral artery. Mean arterial pressure was maintained at $>60 \mathrm{~mm} \mathrm{Hg}$, and sodium chloride $(0.9 \%)$ was infused if needed. A pulmonary artery thermodilution catheter (CritiCath 7F; Ohmeda GmbH \& Co, Erlangen, Germany) was introduced through the right femoral vein for blood sampling and invasive hemodynamic monitoring (Tables 1 and 2 ). Rectal and blood temperatures and electrocardiograms were monitored as well. A urinary catheter (10 Fr) was placed to monitor urine output, and the infused fluids were recorded to determine fluid balance.

\section{Remote Ischemic Preconditioning}

The RIPC design was identical to that of our previous study. ${ }^{13}$ The 9-cm-wide blood pressure cuff was placed around the left hind limb in the experimental group. The cuff was inflated to $250 \mathrm{~mm} \mathrm{Hg}$ for 5 minutes, followed by a 5-minute deflation (reperfusion). The RIPC consisted of 4 inflation-deflation cycles, and the total duration of RIPC was 40 minutes. Control animals were observed for 40 minutes without RIPC.

\section{Motor-Evoked Potentials Monitoring}

A U-shaped incision was made in the scalp to reveal the skull, and the periosteum was removed. Four wire leads were attached to sterile stainless screws and screwed into the skull. The leads were placed $10 \mathrm{~mm}$ lateral to the sagittal suture, 2 on the left side $(8 \mathrm{~mm}$ anterior and $8 \mathrm{~mm}$ posterior to the coronal suture), and 2 on the right side, respectively. The leads were connected to an electrical stimulator (Cadwell, TCS-1, Cadwell Laboratories, Inc, Kennewick, Wash). To measure cortical motor nerve stimuli, stainless-steel needle electrodes were placed through the skin in both hind limbs. An intraoperative neuromonitoring system, Cadwell Cascade Elite with Cascade software, version 2.6 (Microsoft Corporation, Redmond, Wash), was used in the study. A Cadwell TCS-1 constant-voltage electrical stimulator was used for eliciting transcranial electrical MEPs. The multi-pulse-stimulus characteristics were as follows: train length of 4 , interstimulus interval of 2 milliseconds, and stimulus pulse width of $75 \mu$-seconds.

The MEPs were recorded regularly at the baseline, after the RIPC or sham procedure, during the period of SA occlusion at 1-minute intervals, for a period of 30 minutes, after ischemia at 5-minute intervals, and at every 30 minutes throughout the 4-hour follow-up. The MEP data were analyzed with Cascade software, version 2.6. Peak-to-peak amplitude, onset latency, and difference between peak and onset latency were determined from the MEP data (Figure 1).

\section{Surgical Procedures for Spinal Cord Ischemia}

The fourth and seventh intercostal spaces were exposed from the left anterolateral thoracotomy for the dissection of the left subclavian artery and upper thoracic SAs. The rest of the SAs to the level of the diaphragm were exposed through the left, eleventh intercostal-space incision. Every animal had 9 SAs above the diaphragm.

After the surgery, stable anesthetic conditions and hemodynamics were confirmed before baseline MEP amplitude was recorded. Next, 15 minutes after RIPC (or sham), the prepared left subclavian artery and 9 SAs were ligated and occluded consecutively at 5-minute intervals. The decrease of $50 \%$ in MEP amplitude was considered to represent clinically relevant ischemia and was accomplished in all of the experimental animals used in this study.

\section{Postoperative Management}

Surgical incisions were closed in anatomic layers after SA ligations. After a 4-hour follow-up, the animals were extubated and transferred to a 
TABLE 1. Experimental data

\begin{tabular}{|c|c|c|c|c|c|c|c|c|}
\hline Variable & Baseline & Post RIPC & End of SA cutoff & $1 \mathrm{~h}$ post op & $2 \mathrm{~h}$ post op & $4 \mathrm{~h}$ post op & $P_{\mathrm{g}}$ & $P_{t^{* g} \mathrm{~g}}$ \\
\hline Heart rate (bpm) & & & & & & & .39 & $.047 *$ \\
\hline RIPC & $98(89-111)$ & $125(97-137)$ & $81(73-96)$ & $76(68-89)$ & $86(78-87)^{*}$ & $74(70-87)$ & & \\
\hline Control & $105(88-120)$ & $111(91-118)$ & $81(76-86)$ & $93(86-107)$ & $102(94-108)$ & $92(86-93)$ & & \\
\hline MAP (mm Hg) & & & & & & & .97 & .091 \\
\hline RIPC & $89(88-92)$ & $70(68-75)$ & $78(73-89)$ & $74(69-78)$ & $73(70-76)$ & $64(63-68)$ & & \\
\hline Control & $73(68-93)$ & $75(70-88)$ & $83(78-86)$ & $74(71-76)$ & $72(70-75)$ & $64(61-72)$ & & \\
\hline PA pressure $(\mathrm{mm} \mathrm{Hg})$ & & & & & & & $.77 / .33$ & $.08 / .62$ \\
\hline RIPC & $\begin{array}{c}23(20-24) / 9 \\
(7-11)\end{array}$ & $\begin{array}{c}19(16-23) / 10 \\
(8-11)\end{array}$ & $\begin{array}{l}21(20-23) / 9 \\
(6-10)\end{array}$ & $\begin{array}{l}22(18-23) / 10 \\
(5-10)\end{array}$ & $\begin{array}{l}23(22-25) / 10 \\
(8-11)\end{array}$ & $\begin{array}{l}23(21-25) / 10 \\
(9-11)\end{array}$ & & \\
\hline Control & $\begin{array}{l}21(17-23) / 10 \\
(9-10)\end{array}$ & $\begin{array}{c}22(20-25) / 9 \\
(9-10)\end{array}$ & $\begin{array}{l}23(19-25) / 10 \\
(8-11)\end{array}$ & $\begin{array}{l}22(18-24) / 10 \\
(9-11)\end{array}$ & $\begin{array}{l}22(21-23) / 10 \\
(9-11)\end{array}$ & $\begin{array}{l}23(21-27) / 10 \\
(9-11)\end{array}$ & & \\
\hline $\begin{array}{l}\text { Cardiac index } \\
\qquad \mathrm{mL}^{*} \min ^{-1} * \mathrm{~m}^{-2}\end{array}$ & & & & & & & .23 & .58 \\
\hline RIPC & $4.12(3.81-4.36)$ & $3.30(3.10-3.57)$ & $2.80(2.22-3.01)$ & $2.91(2.36-3.13)$ & $2.94(2.58-3.18)$ & $2.93(2.64-3.15)$ & & \\
\hline Control & $3.96(3.49-4.81)$ & $3.87(3.43-4.08)$ & $3.05(2.55-3.20)$ & $3.42(2.91-3.46)$ & $2.78(2.60-3.97)$ & $3.02(2.23-3.52)$ & & \\
\hline Rectal temperature $\left({ }^{\circ} \mathrm{C}\right)$ & & & & & & & .18 & $.018 *$ \\
\hline RIPC & $37.8(37.5-38.9)$ & $37.7(37.1-38.2)$ & $37.3(37.2-38.2)$ & $37.3(37.1-38.4)$ & $37.3(37.1-37.6)^{*}$ & $37.1(36.5-37.6)^{*}$ & & \\
\hline Control & $37.7(37.5-39.0)$ & $38.0(37.3-39.1)$ & $38.4(37.0-38.9)$ & $38.6(37.4-39.1)$ & $38.9(37.9-39.1)$ & $38.7(37.5-39.5)$ & & \\
\hline Blood temperature $\left({ }^{\circ} \mathrm{C}\right)$ & & & & & & & .45 & .61 \\
\hline RIPC & $37.4(36.8-38.8)$ & $37.6(36.6-39.2)$ & $37.4(36.6-39.2)$ & $37.8(36.7-39.4)$ & $37.5(36.9-38.6)$ & $37.4(36.9-38.1)^{*}$ & & \\
\hline Control & $37.5(37.3-39.0)$ & $37.8(37.5-39.2)$ & $38.2(37.7-39.1)$ & $38.7(38.2-39.6)$ & $39.1(38.3-39.4)$ & $38.7(38.3-39.5)$ & & \\
\hline CVP (mm Hg) & & & & & & & .37 & .39 \\
\hline RIPC & $1.0(1.0-2.0)$ & $1.0(0.0-1.0)$ & $1.0(1.0-2.0)$ & $1.0(0.0-2.0)$ & $1.0(0.0-2.0)$ & $1.0(0.0-2.0)$ & & \\
\hline Control & $2.0(0.50-2.5)$ & $1.0(0.50-1.5)$ & $1.0(1.0-3.0)$ & $2.0(1.0-2.5)$ & $2.0(1.0-2.5)$ & $1.5(0.0-3.0)$ & & \\
\hline $\begin{array}{l}\mathrm{O}_{2} \text { consumption } \\
\quad \mathrm{mL} * \mathrm{~kg}^{-1} * \mathrm{~min}^{-1}\end{array}$ & & & & & & & .81 & .48 \\
\hline RIPC & $17.3(14.9-18.1)$ & $15.0(13.9-17.7)$ & $14.6(13.0-18.7)$ & $14.3(13.2-16.4)$ & $15.7(13.4-16.9)$ & $14.6(12.8-15.2)$ & & \\
\hline Control & $15.6(15.0-17.3)$ & $14.7(12.0-18.1)$ & $16.1(15.1-18.4)$ & $14.1(13.3-17.9)$ & $16.5(14.7-17.8)$ & $14.9(14.7-16.5)$ & & \\
\hline $\begin{array}{l}\mathrm{O}_{2} \text { delivery } \\
\quad \mathrm{mL}^{*} \mathrm{~kg}^{-1} * \mathrm{~min}^{-1}\end{array}$ & & & & & & & .74 & .88 \\
\hline RIPC & $54.9(54.3-55.8)$ & $45.5(44.2-50.1)$ & $36.7(31.7-42.7)$ & $39.2(32.2-42.2)$ & $37.5(33.6-44.4)$ & $38.4(36.6-40.3)$ & & \\
\hline Control & $59.4(47.6-63.4)$ & $48.4(46.1-58.0)$ & $38.5(34.0-39.9)$ & $43.7(34.5-47.0)$ & $41.8(35.0-47.5)$ & $37.5(31.3-49.1)$ & & \\
\hline $\mathrm{O}_{2}$ extraction $\mathrm{mL} / \mathrm{dL}$ & & & & & & & .80 & .43 \\
\hline RIPC & $4.0(3.7-4.2)$ & $4.4(4.2-4.9)$ & $5.8(5.4-6.3)$ & $5.0(4.6-5.2)$ & $5.2(5.0-5.7)$ & $4.9(4.5-5.3)$ & & \\
\hline Control & $3.9(3.5-4.6)$ & $3.6(3.1-4.5)$ & $5.5(5.1-5.9)$ & $5.3(4.1-5.7)$ & $5.2(4.7-6.1)$ & $4.9(4.2-7.5)$ & & \\
\hline
\end{tabular}

RIPC $\mathrm{n}=10$. Control $\mathrm{n}=8$. Values are shown as medians and 25th and 75th percentiles. RIPC, Remote ischemic preconditioning; $S A$, segmental artery; post op, postoperatively; $M A P$, mean arterial pressure; $P A$, pulmonary artery; $C V P$, central venous pressure.

recovery room. Analgesia with buprenorphine, given at $6 \mu \mathrm{g} / \mathrm{kg}$ intramuscularly was maintained until the end of the experiment. Neurologic assessment was performed precisely 24 hours after the onset of spinal cord ischemia, using a Tarlov score, as follows: $0=$ spastic paraplegia, no movements; 1 = paraparesis, slight movements; 2 = paraparesis, powerful movements in hind limbs but not able to stand; $3=$ able to stand but unable to walk; 4 = full recovery, normal walking function. ${ }^{14,15}$

The animals were sacrificed with intravenous pentobarbital $(60 \mathrm{mg} / \mathrm{kg})$, and thoracic and lumbar spinal cords were harvested and immersed in $10 \%$ neutral formalin for 2 weeks. The spinal cord was sampled from 5 predetermined areas: thoracic vertebrae nerve roots ${ }^{14} 1-3,4-6,7-9$, and 10-13 and lumbar vertebrae nerve roots 5 1-4. The length of thoracic or lumbar spinal column varied by 1 vertebra among the animals. These samples were then stained with a hematoxylin-eosin stain and scored blind by an experienced neuropathologist.

The sample preparation was performed with a method similar to the one we have used previously. ${ }^{13}$ Hematoxylin-eosin scoring was based on the presence of edema (0-3), hemorrhage (0-2), neuron degeneration (0-1), and the presence of infarcted tissue (0-3). The maximum value of a single region was 9 , and therefore all 5 regions analyzed had a total score range of 0 to 45 .

\section{Biochemical Data}

Blood gas values, $\mathrm{pH}$, electrolytes, plasma lactate levels, serum ionized calcium, glucose, hematocrit, and hemoglobin levels were measured using i-STAT Analyzer (i-STAT Corporation, East Windsor, NJ) at baseline, at 15 minutes after the end of RIPC, immediately after the ninth segmental artery occlusion, and 1 hour, 2 hours, and 4 hours after the ninth segmental artery occlusion (Figure 2).

\section{Statistical Analysis}

Statistical analysis was performed using SPSS (version 22.0; SPSS, Inc, Chicago, Ill) and SAS (version 9.2; SAS Institute, Cary, NC) statistical software packages. Continuous and ordinal variables are expressed as the median and 25th and 75th percentiles in Tables 1 and 2 and Figures 3-5. The repeated measurements were analyzed using a linear mixed model with animals fitted as random, and the best covariance pattern was chosen 
TABLE 2. Metabolic data

\begin{tabular}{|c|c|c|c|c|c|c|c|c|}
\hline Variable & Baseline & Post RIPC & End of SA cutoff & $1 \mathrm{~h}$ post op & 2 h post op & $4 \mathrm{~h}$ post op & $\boldsymbol{P}_{\mathrm{g}}$ & $\boldsymbol{P}_{\mathbf{t}^{* \mathrm{~g}}}$ \\
\hline $\mathrm{pH}$ & & & & & & & .62 & .65 \\
\hline RIPC & 7.48 (7.47-7.49) & $7.49(7.49-7.52)$ & $7.47(7.47-7.50)$ & 7.45 (7.43-7.49) & $7.45(7.44-7.48)$ & $7.47(7.45-7.49)$ & & \\
\hline Control & $7.49(7.48-7.50)$ & $7.48(7.45-7.52)$ & $7.46(7.45-7.50)$ & 7.47 (7.44-7.48) & $7.47(7.42-7.48)$ & $7.45(7.40-7.48)$ & & \\
\hline $\mathrm{PaCO}_{2}(\mathrm{kPa})$ & & & & & & & .92 & .85 \\
\hline RIPC & $5.75(5.63-5.84)$ & $5.52(5.11-5.70)$ & $5.45(5.27-5.56)$ & $5.66(5.26-5.7)$ & $5.34(5.27-5.76)$ & $5.42(5.16-5.72)$ & & \\
\hline Control & $5.64(5.28-5.73)$ & $5.41(5.26-5.54)$ & $5.55(5.33-5.74)$ & $5.48(5.41-5.97)$ & $5.30(5.30-5.72)$ & $5.47(5.27-5.80)$ & & \\
\hline $\mathrm{PaO}_{2}(\mathrm{kPa})$ & & & & & & & .51 & .71 \\
\hline RIPC & $40.0(35.9-42.1)$ & $41.2(38.4-43.5)$ & $40(39.5-41.4)$ & $42.3(40.2-43.7)$ & $41.5(41.4-42.8)$ & $43.3(38.8-44.9)$ & & \\
\hline Control & $38.8(37.2-39.9)$ & $39.5(38.8-44.9)$ & $39.3(36.9-41.4)$ & $40.9(38.2-41.7)$ & $40.9(36.7-42.8)$ & $39.0(31.7-43.1)$ & & \\
\hline Hematocrit $(\%)$ & & & & & & & .21 & .53 \\
\hline RIPC & $28.0(26.0-29.0)$ & $29.0(28.0-30.0)^{*}$ & $28.0(28.0-30.0) \dagger$ & $27.0(24.0-28.0)$ & $26.0(25.0-28.0)$ & $26.0(25.0-26.0)$ & & \\
\hline Control & $28.0(26.0-31.0)$ & $26.0(24.5-27.0)$ & $25.0(24.5-27.5)$ & $26.0(23.0-27.5)$ & $28.0(24.0-28.0)$ & $26.5(23.0-28.0)$ & & \\
\hline Hemoglobin $(\mathrm{g} / \mathrm{L})$ & & & & & & & .20 & .54 \\
\hline RIPC & $95(88-99)$ & $99(95-102)^{*}$ & $95(95-102) \dagger$ & $92(82-95)$ & $88(85-95)$ & $88(85-88)$ & & \\
\hline Control & $95(87-104)$ & $88(84-92)$ & $85(84-94)$ & $88(78-94)$ & $95(82-96)$ & $90(78-95)$ & & \\
\hline Venous glucose $(\mathrm{mmol} / \mathrm{L})$ & & & & & & & .82 & .28 \\
\hline RIPC & $6.0(5.4-6.4)$ & $5.7(4.5-7.6)$ & $3.6(3.4-4.8)$ & $4.4(2.3-7.0)$ & $4.7(3.6-5.9)$ & $3.3(2.9-4.0)$ & & \\
\hline Control & $5.5(1.5-6.9)$ & $5.3(5.0-6.2)$ & $4.2(3.2-5.0)$ & $2.6(1.8-3.8)$ & $5.0(2.5-7.9)$ & $3.9(3.4-5.8)$ & & \\
\hline Venous lactate $(\mathrm{mmol} / \mathrm{L})$ & & & & & & & .30 & .11 \\
\hline RIPC & $0.81(0.66-0.89)$ & $0.59(0.54-0.68)$ & $0.79(0.59-0.95)$ & $1.05(0.67-1.12)$ & $0.81(0.74-1.07) \dagger$ & $0.78(0.67-0.86)$ & & \\
\hline Control & $0.80(0.60-0.99)$ & $0.65(0.45-1.01)$ & $0.62(0.52-0.85)$ & $0.83(0.74-0.98)$ & $1.09(0.98-1.48)$ & $1.16(1.04-1.67)$ & & \\
\hline Osmolality (mmol/kg) & & & & & & & .31 & .65 \\
\hline RIPC & $284(282-286)$ & 285 (285-289) & $283(282-288)$ & $280(276-285)$ & $281(280-287)$ & $282(277-291)$ & & \\
\hline Control & $281(278-284)$ & $283(281-286)$ & $279(279-282)$ & $274(272-279)$ & $283(277-289)$ & $283(278-289)$ & & \\
\hline \multicolumn{9}{|l|}{ Fluid balance $(\mathrm{mL}) \ddagger$} \\
\hline RIPC & $600(400-900)$ & $600(500-738)$ & $850(525-1025)$ & $900(600-1088)$ & $1150(725-1215)$ & $1200(825-1325)$ & .43 & .86 \\
\hline Control & $500(350-750)$ & $500(480-770)$ & $600(600-800)$ & $800(600-800)$ & $850(800-880)$ & $1050(900-1050)$ & & \\
\hline
\end{tabular}

RIPC $\mathrm{n}=10$. Control $\mathrm{n}=8$. Values are shown as medians and 25th and 75th percentiles. RIPC, Remote ischemic preconditioning; SA, segmental artery; post op, postoperatively. $* P<.05$ at single timepoint. $\dagger P>.05$ and $<.06$. $\ddagger$ The fluid balance is presented as a cumulative amount of fluids within the follow-up. The urinary collection bag was drained at the baseline.

according to Akaike's information criteria. Complete independence was assumed across animals (by random statement). Reported $P$ values are as follows: $P$ between groups $\left(P_{\mathrm{g}}\right)$ indicates a level of difference between the groups, $P$ time $\cdot$ group $\left(P_{\mathrm{t}} \cdot \mathrm{g}\right)$ indicates behavior between the groups over time. The Student $t$ test or Mann-Whitney $U$ test was used, as appropriate, to assess the distribution of variables between the study groups. Twotailed significance levels are reported; $P<.05$ was considered statistically significant.

Due to the individual conduction velocities of the spinal cord, MEPs are presented as relative changes from initial values. Hence, the $P$ values of multiple testing $\left(P_{\mathrm{t}} \cdot \mathrm{g}\right.$ and $\left.P_{\mathrm{g}}\right)$ are not reported on MEP values. Their purpose is not for an exact global assessment, but rather as a guide to recognize certain trends between groups at different timepoints.

\section{RESULTS \\ Mortality}

Two experiments were terminated because of intraoperative bleeding and excluded from the analysis. Four

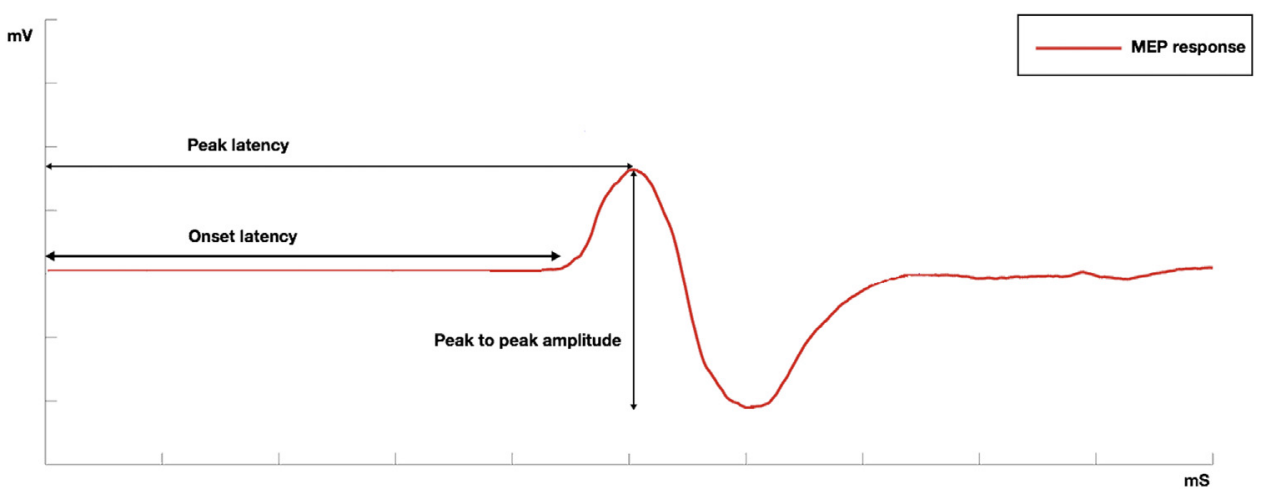

FIGURE 1. Motor-evoked potential response. The figure is modeled from 1 pig. The definitions of the onset latency, peak latency, and peak-to-peak amplitude are shown as lines with arrows. MEP, Motor-evoked potential. 


\begin{tabular}{|c|c|c|c|c|}
\hline \multicolumn{2}{|c|}{$0 \mathrm{~min}$} & $105 \min$ & $\min$ & $\min$ \\
\hline Baseline & RIPC (CTRL sham) & FLUSH 15 min+cutoff SA & 30 min intensive monitoring & Follow-up until $4 \mathrm{~h}$ \\
\hline
\end{tabular}

FIGURE 2. A simplified overview of the protocol. Baseline included anesthesia induction, invasive hemodynamic monitoring, thoracotomy, configuration of baseline MEP, and measurements. Before the start of spinal cord ischemia there was a 15-minute interval (FLUSH) for the data collection after RIPC and surgical preparation. Blood samples were taken at several timepoints, indicated by upward arrows. CTRL, Control; RIPC, remote ischemic preconditioning; $S A$, segmental artery.

animals were lost during observation, owing to respiratory problems. A total of 18 animals survived the 4-hour follow-up (10 in the RIPC group; 8 in the control group), and 14 animals survived at 24-hour follow-up (7 in each group).

\section{Comparability of Study Groups}

The mean weight of the RIPC group was $20.3 \mathrm{~kg}$, and the control group $21.7 \mathrm{~kg}$, respectively $(P=.083)$. Blood $\left(P_{\mathrm{g}}=.45\right)$ and rectal $\left(P_{\mathrm{g}}=.18\right)$ temperatures were similar in the 2 groups. Hemoglobin did not differ between groups $\left(P_{\mathrm{g}}=.20\right)$. No difference was found in mean arterial pressure throughout the entire experiment $\left(P_{\mathrm{g}}=.78\right)$.
Experimental and metabolic data are summarized in Tables 1 and 2.

\section{Motor-Evoked Potentials}

The MEP amplitude change in the RIPC group demonstrated a tendency toward better function of the spinal cord after occlusion of the left subclavian artery and SAs (Figures 3 and 4). Strikingly, the RIPC itself caused an increase in MEP before the onset of spinal cord ischemia. Overall, the difference between groups reached statistical significance in the right hind limb recording at several points in time; left hind limb recordings showed a similar trend.

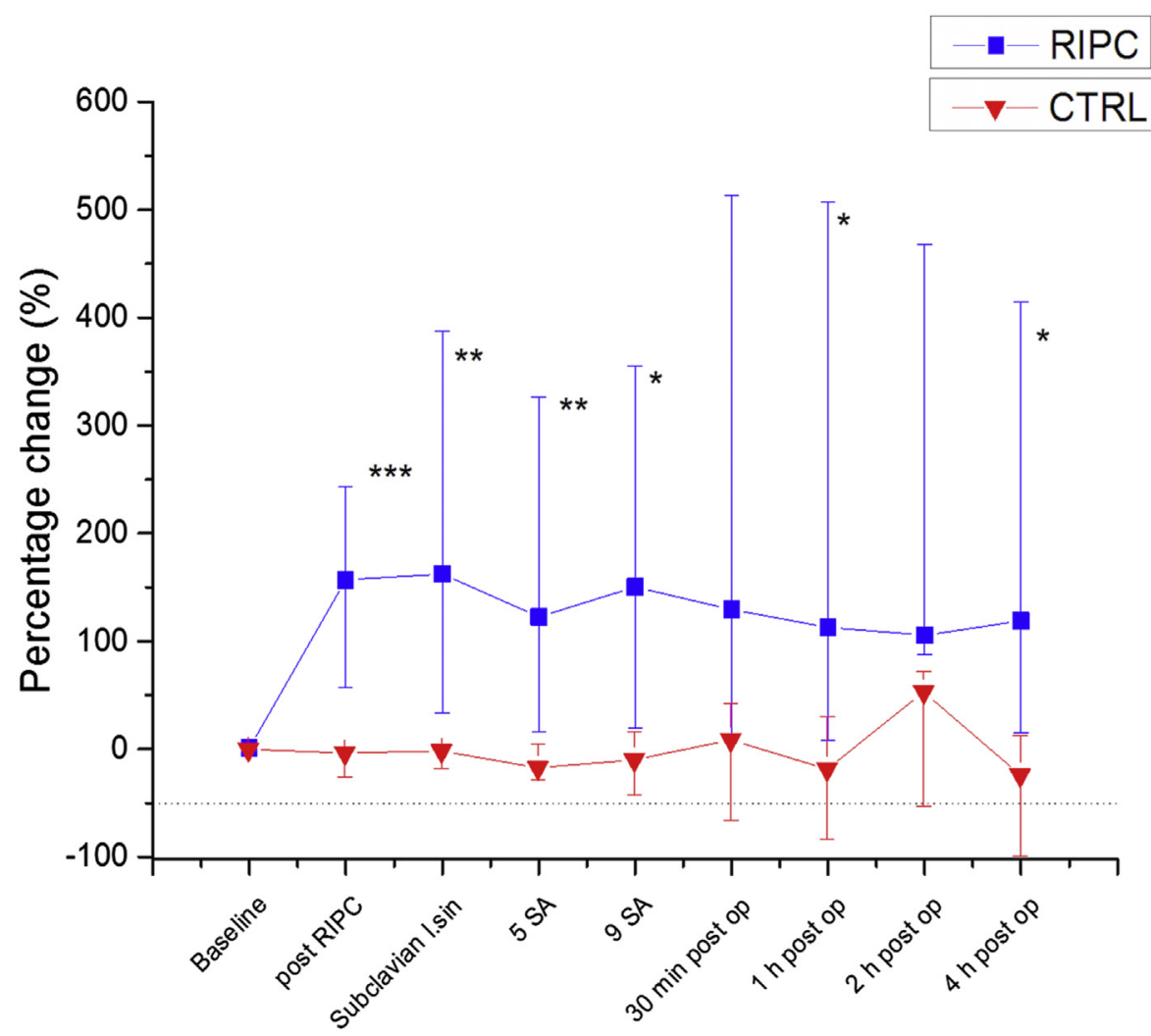

FIGURE 3. Peak-to-peak amplitude of right hind limb. Measurements are compared to the baseline value of 0 . A clinically significant $50 \%$ decrease in the amplitude is marked with a dotted line. The amplitude change starts to diminish steadily after ligation of the left subclavian artery in the control group, whereas the amplitude is higher in the RIPC group after remote ischemic preconditioning. Values represent medians; error bars represent the interquartile range. RIPC, Remote ischemic preconditioning; CTRL, control; Subclavian l.sin, ligation of left subclavian artery; 5 SA, ligation of 5th segmental artery; 9 $S A$, ligation of 9 th segmental artery; post op, postoperatively. $* P<.05 ; * * P<.01 ; * * P<.001$ at a single timepoint. 


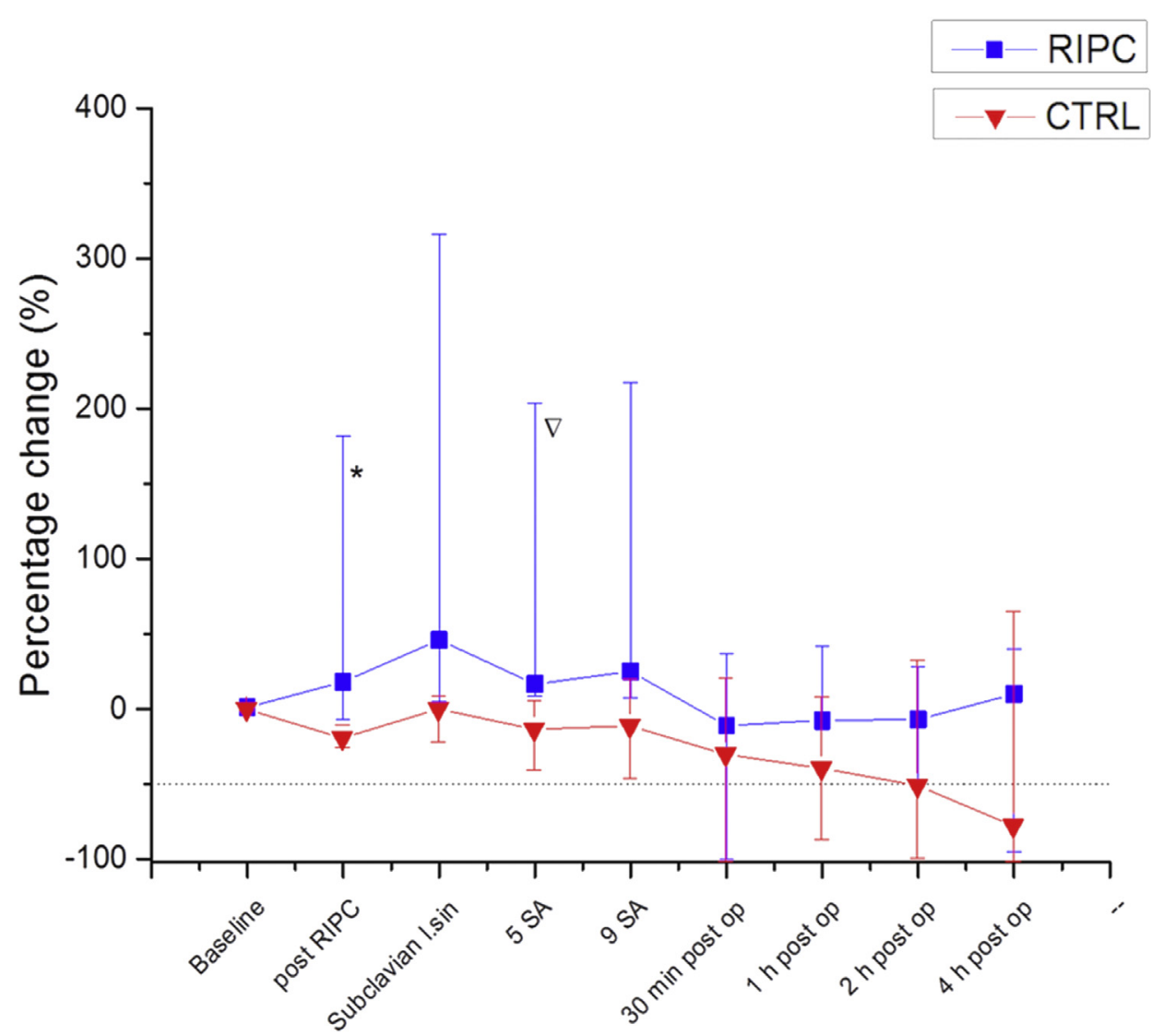

FIGURE 4. Peak-to-peak amplitude in the left hind limb. Measurements are compared to a baseline value of 0 . A clinically significant $50 \%$ decrease in the amplitude is marked with a dotted line. The difference is statistically significant after RIPC. Overall, the trend shows a slightly better preservation of the spinal cord in the RIPC group after ischemia. Values represent medians; error bars represent the interquartile range. RIPC, Remote ischemic preconditioning; CTRL, control; Subclavian l.sin, ligation of left subclavian artery; 5 SA, ligation of 5th segmental artery; 9 SA, ligation of 9th segmental artery; post op, postoperatively. $* P<.05 ; \nabla P<.06$ at a single timepoint.

Onset latency in the right hind limb was significantly shortened in the RIPC group after RIPC and lasted until the last SA occlusion (Figure 5). No statistically significant differences were found in the left hind limb. The differences between onset and peak latency were not significantly different in either limb.

The amplitude in the right hind limb in the RIPC group lasted throughout the 4-hour follow-up (345 minutes), whereas in the control group, the median time to a $50 \%$ amplitude decrease was 195 minutes (143345 minutes), with a significant difference between groups $(P=.044)$. The left hind limb recording did not show differences between the groups $(P=.271)$.

\section{Histology of Spinal Cord}

The median sum of the histopathologic scores in the RIPC group was 9 (7.5-10.5) and also 9 in the control group (6-11), respectively $(P=.713)$. The main findings were edema $(P=.410)$ and hemorrhage $(P=.598)$; the infarction score was similar between groups $(P=1.0)$. Neuron degeneration did not differ significantly between the groups $(P=1.0)$.

\section{Neurologic Evaluation}

The 14 animals that survived through 24-hour observation were neurologically assessed. The RIPC group had a better mean Tarlov score (2.8) compared with the control group (1.8) after 24 hours of spinal cord ischemia, but with no significant differences $(P=.169)$. One subject from the control group had a Tarlov score of 0; the minimum Tarlov score in the RIPC group was 2.

\section{DISCUSSION}

Despite widely used methods to protect the spinal cord during surgical repair of thoracic and thoracoabdominal aneurysms, a significant risk of paraplegia or paraparesis still exists. ${ }^{5}$ Studies using a porcine model seem to be essential in the investigation of the spinal cord blood supply and the reaction to ischemia. ${ }^{10,15}$ During the last decade, we have observed a neuroprotective effect of 


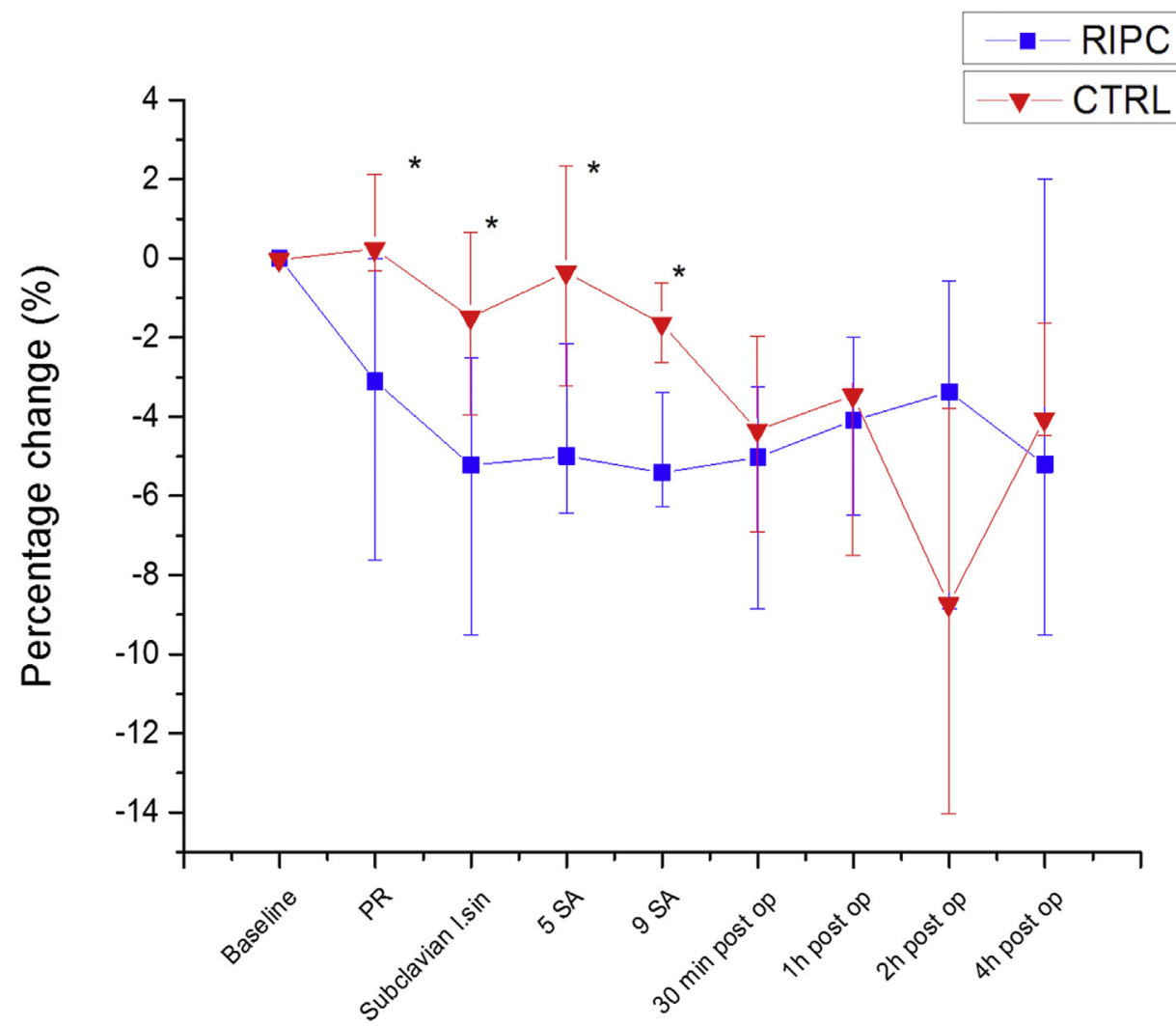

FIGURE 5. Onset latency in the right hind limb. Measurements are compared to a baseline value of 0 . The onset latency is significantly reduced in the RIPC group after intervention until the last segmental artery occlusion. Values represent medians; error bars represent the interquartile range. RIPC, Remote ischemic preconditioning; CTRL, control; $P R$, post-remote ischemic preconditioning; Subclavian l.sin, ligation of left subclavian artery; 5 SA, ligation of 5th segmental artery; $9 \mathrm{SA}$, ligation of 9th segmental artery; post op, postoperatively. $* P<.05$ at a single timepoint.

RIPC in ischemic brain injury associated with hypothermic circulatory arrest. ${ }^{13,16}$ However, evidence has been found against the protective effect, when the RIPC is performed in children suffering from chronic hypoxia. ${ }^{17}$ In this context, the chronic hypoxia may cover the effect of RIPC. In the present study, we established an experimental model and observed that RIPC seems to have protective effects against spinal cord ischemia after occlusion of SAs simulating thoracoabdominal aortic aneurysm surgery (Figures 3 and 5).
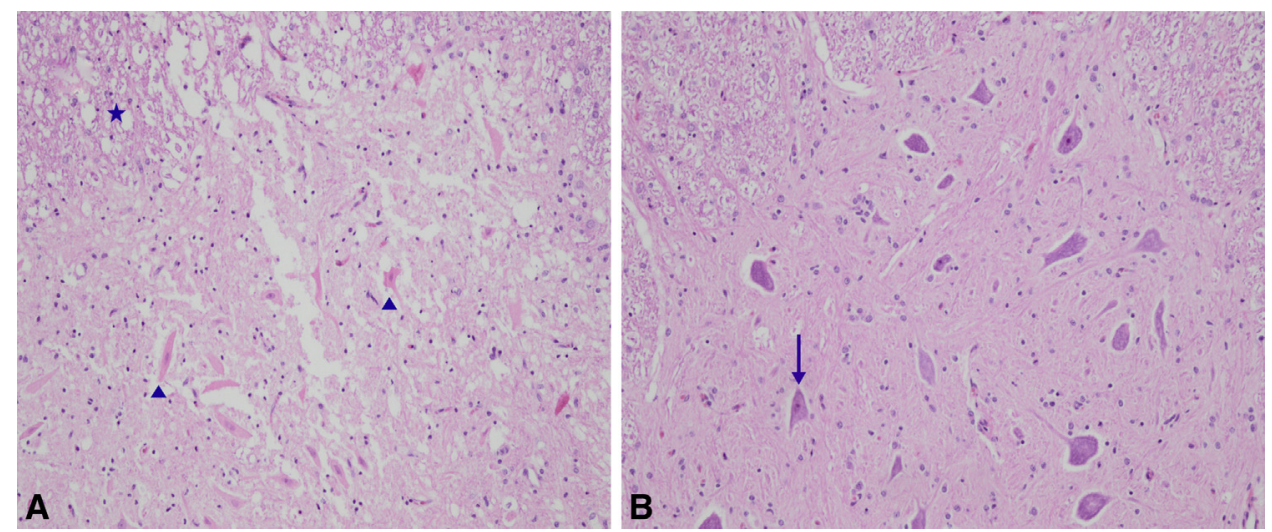

FIGURE 6. Histologic findings from spinal cord. The presented photomicrographs are from ventral horns of the (A) control group porcine and (B) RIPC group porcine. Although the infarction score difference was not significant, the evident ischemia can be seen in the control group porcine. Spinal cord anterior horn shows ischemic, eosinophilic motor neurons (arrowheads), and vacuolar rarefaction of the background (asterisk), whereas in the RIPC group (B), stromal elements and motor neurons (arrow) are viable. 
Recent studies have shown the benefit of RIPC after elective abdominal aorta aneurysm repair, in myocardial and kidney injury, as well as in lung and intestinal dysfunction. $^{18,19}$ The neuroprotective effects of RIPC remain unknown, but preconditioning must be recognized by a cellular sensor that prepares cells for upcoming ischemic stress. Current consensus is that the RIPC neuroprotective effect is mediated by a number of substances, including bradykinin and adenosine. Hence, attenuation of oxidative stress and excitation of the anti-inflammatory signaling pathways seems to provide at least a partial explanation for RIPC protection against spinal cord ischemia. ${ }^{20-22}$ In our previous study, we have found that RIPC reduces adherent leukocytes in cerebral tissue after hypothermic circulatory arrest. ${ }^{23}$ This theory is supported by the large body of the evidence showing the role of inflammation in postischemic neuronal death, in both focal and global ischemia. $^{24}$

In the present study, RIPC improved MEP amplitude significantly in the right hind limb, and the response was more subdued in the left hind limb (Figures 3 and 4). In addition, the onset latency shortened immediately after RIPC in the right hind limb (Figure 5), and the phenomenon was not seen in the left hind limb. Histopathologic findings were minimal, as seen in the sum score, and injuries did not seem to prevent spinal cord function (Figure 6).

The results imply a considerably higher conductivity of motor responses in the spinal cord after RIPC. MEP amplitude is distinctly higher in the RIPC group after intervention, before the initiation of ischemia. The neurons of the spinal cord are better prepared for upcoming ischemia after RIPC, which might be explained by the above-mentioned mechanisms, although they have been studied more extensively in the context of myocardial ischemia. ${ }^{18,25-28}$

In an experimental model by Donato et al. ${ }^{26}$ sectioning of spinal cord extinguished the protective effect of RIPC in the myocardium, indicating the involvement of neuronal afferent signaling. Therefore, we took extra precautions to not damage the femoral nerve while operating on the right femoral vessels. In summary, the mechanisms of RIPC include neurogenic and humoral pathways, and different routes are activated in various organs. However, the reason that MEP amplitude is at least twice as high in the RIPC group cannot be ascertained from our study.

The explanation for the weak left hind limb response might be the sacrifice of the left subclavian artery. ${ }^{15}$ The noncritical number of SAs has been shown ${ }^{15,29}$ to be $9 \pm$ 3. In addition to ligation of the 9 supra-diaphragmatic SAs, the left subclavian was occluded to cause sufficient ischemia to the spinal cord and to lead to interruption of left-side circulation.

This finding supports the theory of a vascular network being responsible for the spinal cord blood supply., ${ }^{5,30}$ Additional support comes from the fact that MEP amplitudes in the control group are more decreased in the left hind limb. Another explanation may be that RIPC caused a reversible peripheral nerve injury to the left hind limb. Irreversible injury is not considered because the neurologic evaluation indicates faster recovery in the RIPC group after 24 hours.

Neurologic status was not significantly better in the RIPC group, although the trend in this group was favorable. The neurologic evaluation was made as early as at the first postoperative day when the animal may still be experiencing the effects of anesthesia and thus cause a bias in neurologic assessment. Moreover, the overall mortality was relatively high in present study. The overnight mortality tended to be higher in the RIPC animals compared with the control animals ( 3 vs 1 ). This difference can be explained by the small group size. On the other hand, the safety of the RIPC method has been demonstrated in many clinical studies. $^{17-19}$

The mean arterial pressure did drop a clinically significant amount in the RIPC group after baseline. The drop is mainly explained as a cause of the transient limb ischemia, but the mean arterial pressure variation stayed inside the safety zone. Thereafter, the hemodynamic conditions remained stable throughout the experiment (Table 1). This stability is important because the spinal-cord perfusion pressure is dependent on the mean arterial pressure, and occlusion of noncritical SAs will become critical if the perfusion pressure decreases. ${ }^{29}$

After the occlusion of the SAs, rectal temperature seemed to be higher in the control group, and as a natural reaction, the heart rate was higher (Table 1). Hyperthermia is known to reduce the latency and increase the conduction velocity of the MEPs; therefore, the differences in rectal temperature do not explain the result. ${ }^{11}$ The higher core temperature might, however, explain the temporary rise in conduction and the reduction in the latency in the control group 2 hours postoperatively (Figures 3 and 5).

\section{CONCLUSIONS}

Our study shows that RIPC in a porcine model preserves spinal cord function after the SAs have been occluded. This study additionally supports the theory that the spinal cord has a vascular network that is responsible for the blood supply. The RIPC could provide a novel strategy to prevent spinal cord ischemic injury. The exact mechanisms of the RIPC neuroprotective effects warrant more studies.

\section{Conflict of Interest Statement}

Authors have nothing to disclose with regard to commercial support.

The authors thank Seija Seljanpera, RN, for her help in caring for the animals, and biostatistician Pasi Ohtonen, MSc, for his expert help with statistical analysis. 


\section{References}

1. Svensson LG, Crawford ES, Hess KR, Coselli JS, Safi HJ. Experience with 1509 patients undergoing thoracoabdominal aortic operations. J Vasc Surg. 1993;17: 357-68; discussion, 368-70.

2. Zoli S, Roder F, Etz CD, Brenner RM, Bodian CA, Lin HM, et al. Predicting the risk of paraplegia after thoracic and thoracoabdominal aneurysm repair. Ann Thorac Surg. 2010;90:1237-44; discussion 1245.

3. Lemaire SA, Price MD, Green SY, Zarda S, Coselli JS. Results of open thoracoabdominal aortic aneurysm repair. Ann Cardiothorac Surg. 2012;1:286-92.

4. Di Luozzo G. Visceral and spinal cord protection during thoracoabdominal aortic aneurysm repair: clinical and laboratory update. J Thorac Cardiovasc Surg. 2013;145(3 Suppl):S135-8.

5. Griepp RB, Griepp EB. Spinal cord protection in surgical and endovascular repair of thoracoabdominal aortic disease. J Thorac Cardiovasc Surg. 2015; 149(2 Suppl):S86-90.

6. Gloviczki P. Surgical repair of thoracoabdominal aneurysms: patient selection, techniques and results. Cardiovasc Surg. 2002;10:434-41.

7. Tapuria N, Kumar Y, Habib MM, Abu Amara M, Seifalian AM, Davidson BR. Remote ischemic preconditioning: a novel protective method from ischemia reperfusion injury—a review. J Surg Res. 2008;150:304-30.

8. de Haan P, Kalkman CJ, Jacobs MJ. Spinal cord monitoring with myogenic motor evoked potentials: early detection of spinal cord ischemia as an integral part of spinal cord protective strategies during thoracoabdominal aneurysm surgery. Semin Thorac Cardiovasc Surg. 1998;10:19-24.

9. Etz CD, Halstead JC, Spielvogel D, Shahani R, Lazala R, Homann TM, et al. Thoracic and thoracoabdominal aneurysm repair: Is reimplantation of spinal cord arteries a waste of time? Ann Thorac Surg. 2006;82:1670-7.

10. Meylaerdts SA, deHaan P, Kalkman CJ, Jaspers J, Vanickey I, Jacobs MJ. Prevention of paraplegia in pigs by segmental artery perfusion during aortic crossclamping. J Vasc Surg. 2000;32:160-70.

11. Pajewski TN, Arlet V, Phillips LH. Current approach on spinal cord monitoring: the point of view of the neurologist, the anesthesiologist and the spine surgeon. Eur Spine J. 2007;16(Suppl 2):115-29.

12. Fulkerson DH, Satyan KB, Wilder LM, Riviello JJ, Stayer SA, Whitehead WE, et al. Intraoperative monitoring of motor evoked potentials in very young children. J Neurosurg Pediatr. 2011;7:331-7.

13. Jensen HA, Loukogeorgakis S, Yannopoulos F, Rimpiläinen E, Petzold A, Tuominen $\mathrm{H}$, et al. Remote ischemic preconditioning protects the brain against injury after hypothermic circulatory arrest. Circulation. 2011;123:714-21.

14. Tarlov IM. Spinal cord compression: mechanisms of paralysis and treatment. Springfield, Ill: Charles C Thomas; 1957.

15. Strauch JT, Spielvogel D, Lauten A, Zhang N, Shiang H, Weisz D, et al. Importance of extrasegmental vessels for spinal cord blood supply in a chronic porcine model. Eur J Cardiothorac Surg. 2003;24:817-24.

16. Yannopoulos FS, Mäkelä T, Niemelä E, Tuominen H, Lepola P, Alestalo K, et al. Improved cerebral recovery from hypothermic circulatory arrest after remote ischemic preconditioning. Ann Thorac Surg. 2010;90:182-8.
17. Jones BO, Pepe S, Sheeran FL, Donath S, Hardy P, Shekerdemian L, et al Remote ischemic preconditioning in cyanosed neonates undergoing cardiopul monary bypass: a randomized controlled trial. J Thorac Cardiovasc Surg. 2013;146:1334-40.

18. Ali ZA, Callaghan CJ, Lim E, Ali AA, Nouraei SA, Akthar AM, et al. Remote ischemic preconditioning reduces myocardial and renal injury after elective abdominal aortic aneurysm repair: a randomized controlled trial. Circulation. 2007;116(11 Suppl):198-105.

19. Li C, Li YS, Xu M, Wen SH, Yao X, Wu Y, et al. Limb remote ischemic preconditioning for intestinal and pulmonary protection during elective open infrarenal abdominal aortic aneurysm repair: a randomized controlled trial. Anesthesiology. 2013;118:842-52.

20. Gurcun U, Discigil B, Boga M, Ozkisacik E, Badak MI, Yenisey C, et al. Is remote preconditioning as effective as direct ischemic preconditioning in preventing spinal cord ischemic injury? J Surg Res. 2006;135:385-93.

21. Dong HL, Zhang Y, Su BX, Zhu ZH, Gu QH, Sang HF, et al. Limb remote ischemic preconditioning protects the spinal cord from ischemia-reperfusion injury: a newly identified nonneuronal but reactive oxygen species-dependent pathway. Anesthesiology. 2010;112:881-91.

22. Bhuiyan MI, Kim YJ. Mechanisms and prospects of ischemic tolerance induced by cerebral preconditioning. Int Neurourol J. 2010;14:203-12.

23. Yannopoulos FS, Arvola O, Haapanen H, Herajärvi J, Miinalainen I, Jensen H, et al. Leg ischaemia before circulatory arrest alters brain leucocyte count and respiratory chain redox state. Interact Cardiovasc Thorac Surg. 2014;18:272-7.

24. Neumar RW. Molecular mechanisms of ischemic neuronal injury. Ann Emerg Med. 2000;36:483-506.

25. Murry CE, Jennings RB, Reimer KA. Preconditioning with ischemia: a delay of lethal cell injury in ischemic myocardium. Circulation. 1986;74:1124-36.

26. Donato M, Buchholz B, Rodríguez M, Pérez V, Inserte J, García-Dorado D, et al Role of the parasympathetic nervous system in cardioprotection by remote hind limb ischaemic preconditioning. Exp Physiol. 2013;98:425-34.

27. Candilio L, Malik A, Ariti C, Barnard M, Di Salvo C, Lawrence D, et al. Effect of remote ischaemic preconditioning on clinical outcomes in patients undergoing cardiac bypass surgery: a randomised controlled clinical trial. Heart. 2014; 101:185-92.

28. Niu X, Zhang J, Chen D, Wan G, Zhang Y, Yao Y. Remote ischaemic conditioning in percutaneous coronary intervention: a meta-analysis of randomised trials. Postepy Kardiol Interwencyjnej. 2014;10:274-82.

29. de Haan P, Kalkman CJ, Meylaerts SA, Lips J, Jacobs MJ. Development of spina cord ischemia after clamping of noncritical segmental arteries in the pig. Ann Thorac Surg. 1999;68:1278-84.

30. Strauch JT, Lauten A, Zhang N, Wahlers T, Griepp RB. Anatomy of spinal cord blood supply in the pig. Ann Thorac Surg. 2007;83:2130-4.

Key Words: remote ischemic preconditioning, motorevoked potentials, spinal cord, cardiovascular procedures 